\title{
Introduction of solar thermal oil circulation pipeline and its numerical simulation
}

\section{Zhen Yang}

School of North China Electric Power University Baoding, Baoding 071000, China; 649066081@qq.com

Abstract: In this chapter, the design of the refrigeration unit with the cooling capacity of $120 \mathrm{~W}$ in the sightseeing beach is used to calculate the lighting area and the collector power of the vacuum tube collector from the solar radiation incident on the solar vacuum tube collector. The design principle of the circulating pipeline of the solar refrigeration system without mechanical power components is introduced. The simplified model of the circulating line of the solar refrigeration system is simulated and analyzed. The flow state parameters of the circulating pipeline are obtained and the feasibility of the theoretical design is verified.

Keywords: heat, Solar collector, Circulation pipeline, Fluent.

\section{Introduction}

The way solar energy is collected is mainly heat collection. Which through the absorption of solar radiation generated by the heat and transfer to the heat transfer medium of solar collectors, is composed of a variety of solar thermal system key components in China's long-term shortage of energy and sustainable development of the current strategy, The state has been the development and utilization of solar energy into a strategic position to consider.

\section{2.solar collector design}

\subsection{Introduction to solar collectors}

The design of the tank vacuum tube type solar collector, the opening for the semi-circular, as shown in Figure 3.1

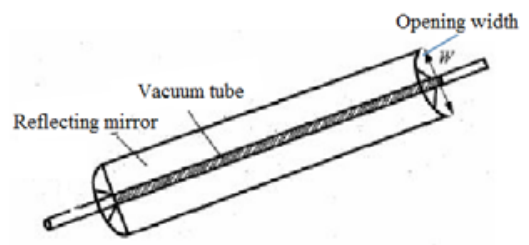

Fig1 Trough type vacuum tube type solar heat collector

\subsection{Determination of vacuum tube power of solar collectors}

1. Single vacuum tube collector area calculation:

Designed according to the current size of the vacuum tube on the market size, combined with the actual operation of the occupied space

Area and other factors, the opening width is the semicircle diameter, slot-type vacuum tube collector specific specifications are as follows: 
Opening width $\quad \mathrm{W}_{\mathrm{a}}=250 \mathrm{~mm}$

Length $\quad \mathrm{L}_{\mathrm{a}}=1550 \mathrm{~mm}$

Vacuum tube diameter $\quad \mathrm{D}_{\mathrm{a}}=47 \mathrm{~mm}$

The efficiency of the trough vacuum tube collector depends on the area of reference, ie, the area of the heat absorber, the area of light, and the total area. In this paper, the lighting area of the collector area is calculated and calculated. The lighting area of the trough collector is the maximum projected area of the solar radiation entering the collector. The design calculates the maximum cross-sectional area of the collector when the sunlight is vertically irradiated to the collector. For the trough collector The length of the vacuum tube with the length of the entire collection tank can be regarded as equal length, then a single vacuum tube collector lighting area:

$\mathrm{A}_{\mathrm{a}}=\mathrm{L}_{\mathrm{a}} * \mathrm{~W}_{\mathrm{a}}$

The solar radiation received by a single vacuum tube is

$\mathrm{I}_{\mathrm{a}}=\mathrm{I}^{*} \mathrm{~A}_{\mathrm{a}}$

The input power of a single vacuum tube is:

$\mathrm{P}_{0}=\mathrm{I}_{\mathrm{a}} * 0.4$

\section{Solar thermal oil circulation pipeline}

\subsection{Solar energy refrigeration system heat source circulation pipe medium selection}

As the heat transfer oil compared to other media (such as water, alcohol) can be recycled at high temperatures, can withstand the diffusion - absorption refrigeration unit start-up temperature. So the design of the choice of thermal oil for recycling. At present, the best application temperature of domestic and foreign economic and practical heat-conducting oil heating is $100{ }^{\circ} \mathrm{C} \sim 300{ }^{\circ} \mathrm{C}$, which is very similar to the working temperature of ammonia-water diffusion-absorption refrigeration unit generator. Heat conduction oil circulation heating is a closed cycle process. The heat transfer oil cools during the circulation and utilizes the heat energy released by the heat transfer to achieve the purpose of heating. The recirculation heating can not only run at lower pressure, but also easily reach the process temperature. Ammonia water cooling unit generator operating temperature of $110{ }^{\circ} \mathrm{C}$ or so, in the heat conduction oil heating conditions, to the generator to provide enough heat. , The design of the choice can be between $12{ }^{\circ} \mathrm{C} \sim 400{ }^{\circ} \mathrm{C}$ for liquid-phase heating system of synthetic heat transfer oil Therminol VP-1 heat transfer oil

\subsection{Principles of Circulation System Design Principles}

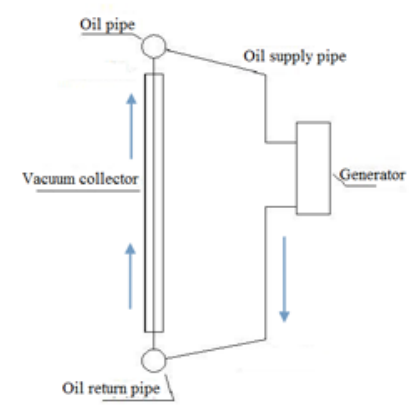

Fig2 Schematic diagram of heat conduction oil circulation system 
Figure 3.2 is the working principle diagram of the gravity cycle heat transfer oil heating system. The single circulation system has an exothermic center (generator) and a heating center (vacuum tube collector). With the supply and return pipe, the solar collector and the cooling The unit generator is connected. Before running, the system is filled with heat transfer oil, heat conduction oil in the vacuum tube collector is heated, the density decreases, while the return from the generator back to the larger density of the oil drive, so that hot oil along the supply pipe Ascending into the generator. In the generator heat oil heat exchange cooling, the density increases, the oil and then return to the vacuum pipe along the return pipe, so as to form the arrow shown in Figure 3.2 flow. In order to simplify the analysis, regardless of the heat transfer oil along the pipeline flow due to the cooling of the wall and the cooling effect, that in Figure 3.2 of the circulating pipe, the heat transfer oil temperature in the vacuum tube collector to maintain high temperature $\mathrm{g}$, in the generator The temperature is reduced to $\mathrm{T}$, the thermal difference between the hot and cold on both sides of the heat caused by the pressure difference is to promote the heat transfer oil in the system circulation of gravity cycle pressure. The pressure of the system is

$\mathrm{P}=\mathrm{gL}(\rho \mathrm{h}-\rho \mathrm{g})$

$\mathrm{P}-$ - The pressure of the circulatory system, $\mathrm{Pa}$;

g-C Gravitational acceleration, $\mathrm{m}^{2} / \mathrm{s}$

L-— vertical distance, $\mathrm{m}$

$\rho \mathrm{h}$ - - Heat transfer oil back to the oil density, $\mathrm{kg} / \mathrm{m}^{3}$

$\rho \mathrm{g}$ - - Heat transfer oil supply density, $\mathrm{kg} / \mathrm{m}^{3}$

Heat-conducting oil pipe selection Seamless steel pipe, diameter of $16 \mathrm{~mm}, \square 30 \mathrm{~mm}$ Select a number of 90 elbow, connected to the generator to facilitate the overall disassembly and transportation.

\section{Numerical Simulation of the Structure of Conductive Oil Circulation System}

Because the structure of the system is more complicated, the test is more complicated and the processing time is long. Therefore, the numerical simulation of the oil circulation system is carried out in this paper.

\section{1 geometric simplification model of the conducting oil circulation system}

Considering that the number of vacuum tubes in the design is more, the flow state in the tube is more complicated and the calculation is more difficult. Therefore, in order to explain the principle, the simplified simulation of the single-tube circulation system is carried out under the theoretical design conditions. Ignore the heat loss due to heat dissipation during the heat transfer process; assuming that the heat transfer oil is uniformly heated in the vacuum tube, the temperature is constant; the solar collector is simplified as a heating surface 


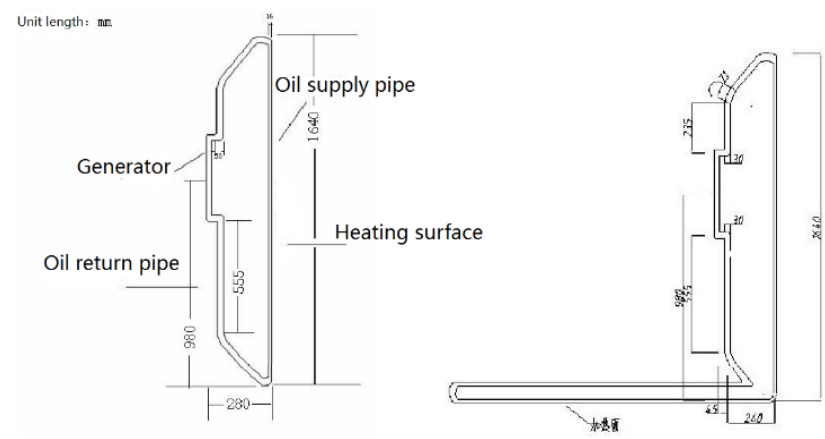

Fig3 Vertical placement Fig4 Horizontal placement

Under the premise of the above assumptions, taking into account the actual installation height of the circulatory system and the size of the refrigerator, this paper presents two models of single-tube circulatory system for conducting oil,. Figure 3 is to put the solar collector vertically, that is, the design style mentioned earlier, Figure 4 is based on the actual structure of some solar collectors, need to be horizontal. The first model of solar collectors due to the use of vertical release type, the installation is more convenient. The second model covers a large area, suitable for large collectors, can be placed on the roof, do not need to use the case of frequent use.

\subsection{FLUENT simulation analysis of single-tube circulation system for heat transfer oil}

At present the international comparison of the popular use of Fluent simulation of fluid, heat transfer and chemical reactions related to the question

question. This section uses Fluent software to simulate the single-tube circulation system of the heat transfer oil

As the solar refrigeration system circulation pipe is simplified as a single oil circulation system, the heat is distributed evenly on the single-tube trough collector surface. For convenience calculation, the heating temperature is $160{ }^{\circ} \mathrm{C}$. It is necessary to compare the energy of the solar radiation to the vacuum tube of the collector and the solar radiation energy absorbed by the vacuum tube of the collector without considering the heat dissipation and heat preservation, and verify the feasibility of the heating temperature in the simulation.

\subsection{Heat conduction oil single tube circulation system simulation results}
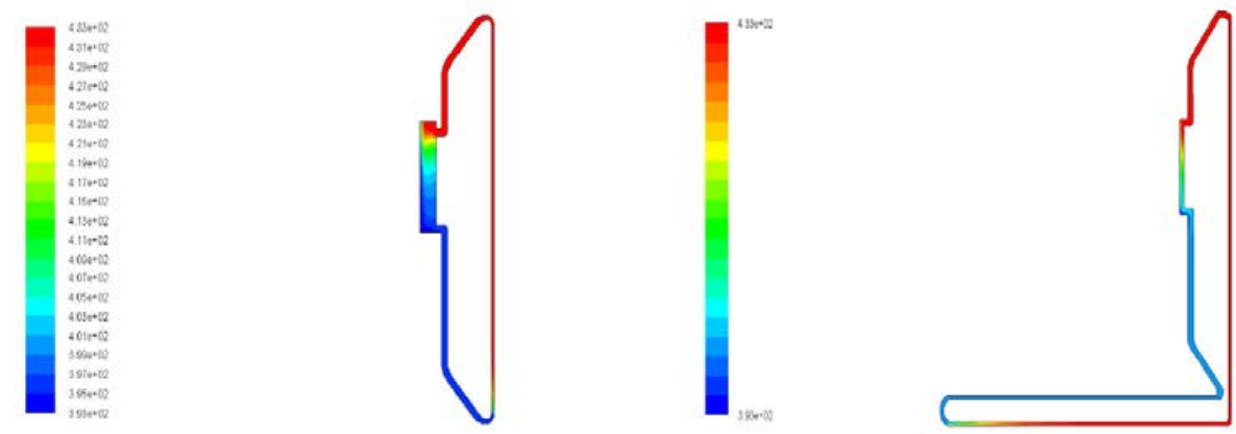

Fig5 Temperature diagram of single pipe circulating system

It can be seen from Figure 5, the two models in the collector heat transfer oil from the low temperature gradually heated to high temperature $433 \mathrm{~K}$, with the temperature rising, through the oil supply pipe, transported to the generator, the heat in the 
generator by ammonia absorption Refrigeration system of refrigerant ammonia continue to absorb, reduce the temperature, and ultimately reduced to $393 \mathrm{~K}$, and finally through the oil return pipe, to reach the solar collector, to complete a cycle.

\section{References}

[1] Zarza.DISS phase II-Final Project Report. EU-Project No. JOR3-CT980277 . 2007

[2] Eickhoff M.Incident angle modifier of the LS-3-DISS collectors. Internal Report of the DISS project . 2002

[3] Applied research concerning the direct steam generation in parabolic troughs[J]. Solar Energy . 2003 